\title{
A Genome-Wide Association Study to Identify Potential Germline Copy Number Variants for Sporadic Breast Cancer Susceptibility
}

\author{
Yadav Sapkota a, b Ashok Narasimhan ${ }^{c}$ Mahalakshmi Kumaran ${ }^{c}$ \\ Badan S. Sehrawat ${ }^{c}$ Sambasivarao Damaraju ${ }^{c, d}$ \\ ${ }^{a}$ Department of Epidemiology and Cancer Control, St. Jude Children's Research Hospital, Memphis, Tenn., USA;

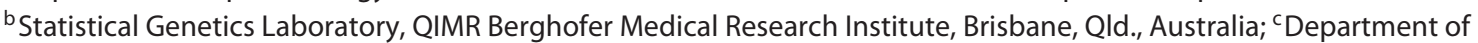 \\ Laboratory Medicine and Pathology, University of Alberta, and ${ }^{\mathrm{d}}$ Cross Cancer Institute, Alberta Health Services, \\ Edmonton, Alta., Canada
}

\section{Key Words \\ Association study · BRCA · Breast cancer susceptibility · Copy number variations · Copy number variation calling algorithm · Genetic predisposition - Germline DNA . Polygenic disease - Single nucleotide polymorphism . Sporadic breast cancer}

\begin{abstract}
Breast cancer $(\mathrm{BC})$ predisposition in populations arises from both genetic and nongenetic risk factors. Structural variations such as copy number variations (CNVs) are heritable determinants for disease susceptibility. The primary objectives of this study are (1) to identify CNVs associated with sporadic BC using a genome-wide association study (GWAS) design; (2) to utilize 2 distinct CNV calling algorithms to identify concordant CNVs as a strategy to reduce false positive associations in the hypothesis-generating GWAS discovery phase, and (3) to identify potential candidate CNVs for follow-up replication studies. We used Affymetrix SNP Array 6.0 data profiled on Caucasian subjects (422 cases/348 controls) to call CNVs using algorithms implemented in Nexus Copy Number and Partek Genomics Suite software. Nexus algorithm identified CNVs associated with BC (731 autosomal CNVs with $>5 \%$ frequency in the total sample and $\mathrm{Q}<0.05$ ). Thirteen CNVs were identified when Partek algorithm-called
\end{abstract}

\section{KARGER}

(C) 2016 S. Karger AG, Basel

E-Mail karger@karger.com

www.karger.com/cgr
CNVs were overlapped with Nexus-identified CNVs; these CNVs showed concordances for frequency, effect size, and direction. Coding genes present within BC-associated CNVs were known to play a role in disease etiology and prognosis. Long noncoding RNAs identified within CNVs showed tissue-specific expression, indicating potential functional relevance of the findings. The identified candidate CNVs warrant independent replication.

(c) 2016 S. Karger AG, Basel

Breast cancer (BC) is the most frequently diagnosed cancer among women worldwide, with more than a million new cases each year. The disease results from the combined effects of genetic, environmental, reproductive, and lifestyle risk factors [Sehrawat et al., 2011; Sapkota et al., 2013a, b]. Single nucleotide polymorphisms (SNPs) identified through genome-wide association studies (GWAS) helped explain only a small proportion of genetic risk in BC [Stacey et al., 2007, 2008; Kidd et al., 2008; Krepischi et al., 2012a; Kuusisto et al., 2013; Long et al., 2013; Michailidou et al., 2013; Sapkota et al., 2013b, 2014]. It is believed that additional variants need to be

Y.S. and A.N. contributed equally to this work.
Dr. Sambasivarao Damaraju, $\mathrm{PhD}$

Department of Laboratory Medicine and Pathology University of Alberta and Cross Cancer Institute, Alberta Health Services 11560 University Avenue, Edmonton, AB T6G 1 Z2 (Canada)

E-Mail sdamaraj@ualberta.ca 
identified to account for the 'missing heritability' [Michailidou et al., 2013]. In addition to SNPs as heritable markers, variations in the germline DNA such as insertions, deletions, and copy number variations (CNVs) are hypothesized to confer genetic susceptibility to complex traits [Feuk et al., 2006; Conrad and Hurles, 2007; McCarroll and Altshuler, 2007; Bailey et al., 2008; Diskin et al., 2009; Conrad et al., 2010b; McCarroll, 2010; Wellcome Trust Case Control Consortium et al., 2010; Masson et al., 2014; Handsaker et al., 2015]. Germline CNVs, therefore, are pursued as potential candidates to explain the missing heritability. This optimism stems from the fact that CNVs occupy a larger portion of the genome compared to SNPs. CNVs are the most common type of structural variations in the human genome and are generally defined as gains or losses of DNA segments $\geq 1 \mathrm{~kb}$. Currently, these cutoffs have been redefined to include microdeletions and amplifications [Conrad and Hurles, 2007; McCarroll and Altshuler, 2007; Conrad et al., 2010a; McCarroll, 2010]. CNVs may exert their phenotypic effects through either gene dosage or cis-acting regulatory elements. Over 400,000 unique CNVs were reported in the Database of Genomic Variants (DGV; http://dgv.tcag.ca/dgv/app/ home) as of 2014 [MacDonald et al., 2014].

Recently, rare CNVs were shown to be important sources for interindividual variations in the susceptibility to familial BC [Gonzalez et al., 2005; Krepischi et al., 2012b; Pylkas et al., 2012; Kuusisto et al., 2013; Masson et al., 2014; Park et al., 2015]. Further, we have demonstrated that copy-neutral losses of heterozygosity are also an important class of structural variations and serve as biomarkers of prognostic value in a BC setting [Sapkota et al., 2013a]. Large CNVs have an appreciable impact on many human diseases, including non-small-cell lung cancer, HIV infection, developmental delay, schizophrenia, autism, and idiopathic learning disability [Glessner et al., 2009, 2010; Hollox and Hoh, 2014; Li et al., 2014]. However, data on the role of structural variations in predisposition to sporadic BC susceptibility is scanty.

Herein, we sought to investigate the role of germline $\mathrm{CNV}$ s in sporadic BC susceptibility using 422 invasive BC cases and 348 healthy individuals from Alberta, Canada, which were genotyped employing Affymetrix GenomeWide Human SNP Array 6.0 [Sapkota et al., 2013a]. Currently, there are no consensus guiding principles on the use of segmentation algorithms, nor ideal SNP or CNV arrays from any manufacturer to identify CNVs associated with disease susceptibility. The catalogues of variants available as a source of reference are of limited utility, and a gold standard reference is still evasive, contrary to the previous attempts to create such a reference database [Kidd et al., 2008; Korn et al., 2008; Conrad et al., 2010b; Zhang et al., 2011]. High-throughput methods are not available to validate these signatures in a cost-effective manner, and qRT-PCR is one suggested approach to select a number of targets for a limited sample size [Lin et al., 2011]. Keeping these limitations in mind, several recent studies made recommendations to use more than 1 CNV calling algorithm to ascertain CNV calls [Lin et al., 2011; Zhang et al., 2011]. We therefore designed our study to include $2 \mathrm{CNV}$ calling algorithms, Partek Genomics Suite 6.6 (Partek GS) and Nexus Copy Number 6.0 (Nexus CN), respectively. The rationale for the use of 2 algorithms was to overcome inherent constraints by the individual algorithms in ascertaining the breakpoints, an otherwise tedious task, and to enable identification of overlapping CNVs [Conrad et al., 2010a]. Our emphasis is also on identifying common CNVs as heritability determinants. Common CNVs are defined as those which are present in frequencies of $>5 \%$ in the total cohort (cases and controls together).

The comparisons between algorithms potentially identify concordant signatures. Concordance criteria are defined as CNVs with (1) 100\% region overlap between Nexus and Partek, (2) similar copy number status, and (3) effect size in a similar direction (gain or loss). To our knowledge, this is the first study in CNV-GWAS for BC to apply stringent filtering criteria of $100 \%$ concordance by the above selected criteria. Thus, the present study offers a novel premise hitherto unexplored to identify common CNVs as genetic susceptibility determinants in BC. Exploring the possible functional significance of the identified CNVs may offer insights into the etiology of the disease and provide potential loci for replication in independent studies.

\section{Materials and Methods}

\section{Study Participants}

Cases $(n=422)$ included in this study were predominantly of sporadic BC (defined by age of onset), non-metastatic at the time of diagnosis, and were accessed from the provincial tumor bank, the Alberta Cancer Research Biobank (formerly Canadian Breast Cancer Foundation, CBCF, Tumor Bank) located at the Cross Cancer Institute, Edmonton, Alta., Canada (http://www.acrb.ca/ about-us/). The tumor bank database also provided clinicopathological information of the stored samples. A detailed description of the bank and the specimens used in this study has been given elsewhere [Sehrawat et al., 2011; Sapkota et al., 2013a, b]. The cases had a pathologically confirmed diagnosis of invasive BC and were predominantly characterized by the late onset of the disease ( $>90 \%$ of the cases were aged $40+$ years at the time of diagnosis;
Identification of Germline CNVs for Breast Cancer Susceptibility
Cytogenet Genome Res 2016;149:156-164 DOI: $10.1159 / 000448558$ 
table 1$)$. The controls $(n=348)$ used in this study were healthy women (cancer free with no documented family history of cancer at the time of recruitment) from the same geographic location (Alberta, Canada). The controls were accessed from the Tomorrow Project (http://in4tomorrow.ca), a prospective cohort study which aims to collect DNA and information about health and lifestyle factors of $\sim 50,000$ healthy Albertans to understand the etiology of complex diseases, including cancers and cardiovascular diseases [Sehrawat et al., 2011; Sapkota et al., 2013a, b]. The controls were approximately frequency matched to cases based on a 10-year age group, with $>86 \%$ of the controls aged $40+$ at the time of recruitment in the study. Both cases and controls were predominantly of Caucasian origin.

\section{DNA Extraction and Genotyping}

Germline DNA was extracted from peripheral blood samples of both cases and controls, using commercially available Qiagen ${ }^{\mathrm{TM}}$ (Mississauga, Ont., Canada) DNA isolation kits, as described earlier [Sehrawat et al., 2011; Sapkota et al., 2013a]. Whole genome genotyping was carried out using the Affymetrix Genome-Wide Human SNP Array 6.0, following the guidelines provided by the manufacturer. CNV signal intensity data was acquired directly from 'CEL' files by using data import functionality of Partek GS and Nexus CN. Genotype data was acquired by using a default Affymetrix genotyping algorithm (Birdseed v2). Sample qualities were assessed by contrast quality control (CQC), a measure of performance of genotyping experiments recommended by the manufacturer. All samples had CQC $>2.0$; a CQC $\geq 1.7$ is considered acceptable [Sehrawat et al., 2011].

\section{Population Stratification}

We used the principal component analysis-based Eigenstrat method to control for potential confounding effects of population stratification, using SNP genotype data of the 770 samples (cases and controls), as described earlier [Sehrawat et al., 2011; Sapkota et al., 2013a]. Publicly available genotype data of the 270 HapMap samples was used as a reference to infer the genetic ancestry of the samples. We detected 84 individuals ( 56 cases and 28 controls) as non-Europeans, using $\geq 3$ standard deviations from the top 2 principal components (online suppl. fig. 1; for all online suppl. material, see www.karger.com/doi/10.1159/000448558). These ancestry outliers were removed, leaving 366 cases and 320 controls for the downstream analysis. Cases and controls employed in the study showed no cryptic relatedness and were detected by assessing the identity by descent (IBD) estimates; all pair-wise IBD correlations were $<0.25$, as described earlier [Purcell et al., 2007].

\section{Workflow for Identification and Analysis of CNVs Using}

Nexus CN 6.0

Intensity files of the 270 HapMap samples were used as a reference/baseline to calculate $\log _{2}$ ratios and B-allele frequencies for each sample, followed by quantile normalization. Probe-to-probe variance was calculated and used to identify extreme outliers due to copy number breakpoints. Using a default value of $3 \%$ for outlier removal, which is $1.5 \%$ of probes each on the top and bottom of the list of total probes, we removed 6 cases showing higher CQC scores vis-à-vis elevated noise to signal ratios, leaving 360 cases and 320 controls (total $n=680$ ) for the final analysis. SNP-Fast Adaptive States Segmentation Technique 2 (SNP-FASST2) segmentation algorithm, combined with quadratic wave correction imple-
Table 1. Patient characteristics

\begin{tabular}{lcc}
\hline Characteristics & $\begin{array}{c}\text { BC cases } \\
(\mathrm{n}=422)\end{array}$ & $\begin{array}{l}\text { Healthy controls } \\
(\mathrm{n}=348)\end{array}$ \\
\hline Age group $^{\mathrm{a}}$ & & \\
$\quad<40$ years & 39 & 40 \\
$40-50$ years & 129 & 130 \\
$50-60$ years & 153 & 99 \\
$60-70$ years & 64 & 77 \\
$70-80$ years & 29 & 2 \\
$>80$ years & 8 & 0 \\
Median age, years & 52 & 50 \\
Range & $21-90$ & $36-70$ \\
\hline BMI group & & \\
$<18.5$ & 2 & 155 \\
18.5-24.99 & 121 & 177 \\
$25.0-39.99$ & 233 & 12 \\
$>40$ & 23 & 0 \\
Missing & 43 & 25.3 \\
Median BMI & 27.4 & $22.9-29.8$ \\
25-75th percentile & $24.3-31.4$ & \\
\hline
\end{tabular}

a Age was recorded at diagnosis (for cases) and at blood withdrawal (for controls).

mented in Nexus was used to identify CNVs in the 680 samples. The SNP-FASST2 is a Hidden Markov Model-based segmentation algorithm, which uses normalized $\log _{2}$ ratio values of $\sim 1.8$ million SNP and copy number probes in the Affymetrix Genome-Wide Human SNP Array 6.0 to make a CNV call. The statistical significance threshold for a copy number call was set at $5.0 \times 10^{-10}$, with a minimum number of probes per segment of 25 and maximum contiguous probe spacing of $5 \mathrm{~kb}$. $\log _{2}$ ratio values of 0.2 and -0.2 were used to define single copy number gains and losses, respectively, while $\log _{2}$ ratio values of 0.7 and -1.1 were used to define more than 2 copies of gains and losses, respectively. Auto gender correction was applied to call CNVs in X chromosomes.

Workflow for CNV Analysis Using Partek Genomics Suite 6.6

Intensity data from Affymetrix CEL files $(n=686)$ was imported into Partek GS and quantile normalized. Copy number segments were identified using the genomic segmentation algorithm with the following default settings: minimum number of probes per segment $>10$ (referred to as genomic markers by Partek); $\mathrm{p}$ value threshold $=0.001$; signal $/$ noise ratio $=0.3$; diploid copy number $=1.7-2.3$, with copy number loss and gain below and above these thresholds, respectively. The segmentation data obtained was processed using 270 HapMap samples to create the baseline (representing copy neutral state for a diploid genome) as described above for the Nexus platform. Genome assembly from Genome Reference Consortium human (GRCh) build 37 (Human Genome version, hg19) was used as a source to annotate the identified genomic variants. The identified CNVs were also interrogated in DGV, and the percent overlaps of the CNVs with the recent version of DGV were reported (http://dgv.tcag.ca/). 
Fig. 1. Size distribution of CNVRs from Partek signatures relative to Nexus CNVRs.

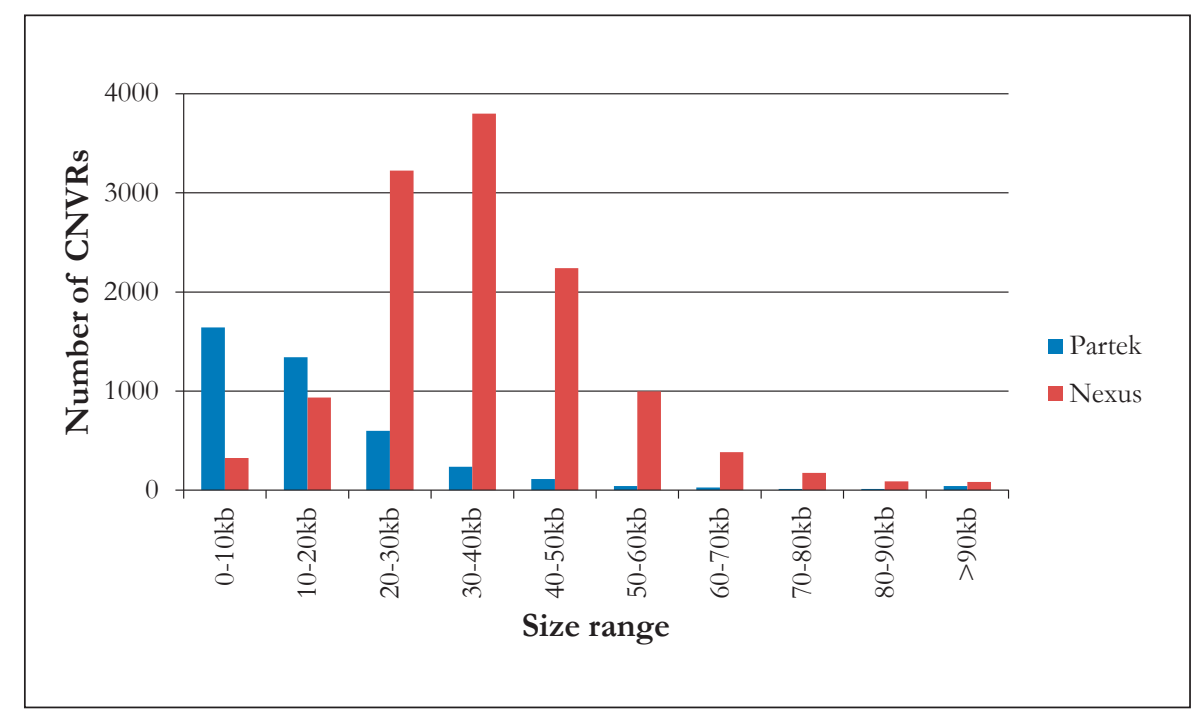

Since the 2 algorithms we used implement their own sample pre-processing and analysis steps, and there is no easier way to standardize these steps for absolute comparisons of algorithm efficiency in identifying the CNVs, the default parameters recommended were used. We therefore made a systematic effort to compare signatures based on criteria of concordances of CNV calls between algorithms (described above). Previous investigators used an arbitrary cut-off of $25-50 \%$ of overlap of the sequence as a criterion for concordance [Lin et al., 2011; Zhang et al., 2011; Castellani et al., 2014]. However, we defined concordance as $100 \%$ overlap of CNV regions to be on the more stringent side to accommodate the mismatch of default parameters for segmentation by individual algorithms. The deviations from less than $100 \%$ of the region overlap, if considered (and as reported by others), would only result in a higher concordance and not less. However, we refrained from such assumptions, and as such many of the identified CNVs in our study or their potential concordances may have been underestimated in our analysis. This is true of any algorithm comparisons implemented thus far for $\mathrm{CNV}$ association analysis, as acknowledged by others [Grayson and Aune, 2011; Lin et al., 2011; Zhang et al., 2011; Cantsilieris et al., 2014; Castellani et al., 2014].

\section{Association of CNVs with BC Susceptibility}

Frequencies of germline CNVs were compared between cases and controls using 2-tailed Fisher's exact test (within Nexus CN) or Pearson's $2 \times 2 \chi^{2}$ test (within Partek GS) to identify CNVs associated $(\mathrm{Q}<0.05)$ with $\mathrm{BC}$ susceptibility. The $2 \times 2$ contingency table compares the frequency of copy number status (loss or gain) between the cases and controls. Correction for multiple comparisons was carried out using the Benjamini-Hochberg false discovery rate and given as a $\mathrm{Q}$ value. We report associations with deletions only or gains only from gene and non-gene regions in a casecontrol setting. We used the 'gap' package [Szatkiewicz et al., 2014] using the R program (https://cran.rproject.org/web/packages/gap/ vignettes/gap.pdf) to calculate the power needed to detect CNVs associated with $\mathrm{BC}$ susceptibility under the following assumptions: additive model, sample size $\mathrm{n}=770$, and life-time risk for $\mathrm{BC}$ at $11 \%$ (1/9 among Caucasians). For a genotype relative risk of 1.5

Identification of Germline CNVs for

Breast Cancer Susceptibility and a risk allele frequency range of $5-10 \%$, the power is $32-52 \%$; for a relative risk of 2 , the estimated power at risk allele frequency of $5-10 \%$ is $77-94 \%$.

\section{Functional Relevance of the BC-Associated CNVs}

$\mathrm{BC}$-associated CNVs may harbor cis-acting elements and potentially regulate the expression of flanking genes [Wu et al., 2012]. Similarly, CNVs may also harbor non-protein-coding genes such as microRNAs (miRNAs), long noncoding RNAs (lncRNAs), etc. We interrogated for miRNA genes embedded within CNV regions (https://genome.ucsc.edu/) and lncRNAs using GENCODE database (http://www.gencodegenes.org/) [Meng et al., 2014; Sorensen et al., 2015]. We used TANRIC (http://ibl.mdanderson.org/tanric/_design/basic/index.html) to gain potential insights into the breast tissue-specific expression of lncRNAs [Li et al., 2015]. TANRIC uses The Cancer Genome Atlas (TCGA) transcriptome data generated on the next-generation sequencing platform from as many as 20 different tumor types including BC.

\section{Results}

\section{Characteristics of the Identified Germline CNVs}

We identified 13,417 CNVs ( 50\% losses and 50\% gains) in Nexus, of which 731 were autosomal CNVs showing association with $\mathrm{BC}(\mathrm{Q}<0.05)$ at frequencies of $>5 \%$ in the sample cohort (online suppl. table 1 ). We identified 20,154 CNVs ( 68\% losses and 32\% gains) using the Partek GS algorithm, of which 7,155 were autosomal and showed association with $\mathrm{BC}(\mathrm{p}<0.05$; data not shown). The CNVs identified by each algorithm had consistent patterns in terms of their mean length across all autosomes. There were no large deviations observed within the algorithm for the mean length of CNVs across 
all chromosomes (online suppl. fig. 2A). However, a comparison of the CNVs identified between independent algorithms (from Nexus CN and Partek GS) revealed several distinct characteristics. The Nexus $\mathrm{CN}$-identified CNVs were larger (mean $=35.86 \mathrm{~kb}$ ) compared to the Partek GS signatures (mean $=3.88 \mathrm{~kb}$ ), and these differences in their mean size are statistically significant ( $t$ test, $\mathrm{p}<0.001$ ) (online suppl. fig. 2A). Also, the numbers of CNVs identified by the individual algorithms were different as also the size range for the identified CNVs (online suppl. fig. 2B). The CNVs identified by Partek GS showed regions of contiguity with flanking $\mathrm{CNVs}$ and as such were merged and designated as copy number variable regions (CNVRs). We compared the CNVRs from Partek GS with Nexus CN CNVs (fig. 1). Partek GS-identified CNVRs were in the range of $1-50 \mathrm{~kb}$, whereas Nexus $\mathrm{CNVs}$ were in the range of 1 to $>90 \mathrm{~kb}$. Because of these differences and owing to the predominantly large size of CNVs identified by Nexus, in our subsequent experiments we mapped the Partek GS signatures to Nexus CN to show concordance of CNVs identified by both algorithms.

\section{Concordant CNVs Identified by Nexus CN and Partek} GS Algorithms

We investigated if the CNVs identified by Nexus CN and Partek GS algorithms would show concordance. The concordant signatures were defined as CNVs with (1) 100\% region overlap between Nexus and Partek, (2) similar copy number status, and (3) effect size in a similar direction. Data in table 2 indicates that 13 of the Nexus CN-identified CNVs showed concordance with Partek GS signatures. Of these, $6 \mathrm{CNVs}$ harbored protein-coding genes, and the rest were in intergenic regions. Overall, the CNVs identified were from 8 distinct chromosomes. The CNV length for concordant signatures ranged from 6.7 to $41.7 \mathrm{~kb}$, and showed a differential frequency between cases and controls in the range of 5-13.75. The concordant CNVs appear to be independent disease risk loci as we did not observe linkage disequilibrium with GWAS-identified SNP loci for BC susceptibility (data not shown).

The odds ratios, i.e., effect sizes for CNVs associated with $\mathrm{BC}$ risk were higher (Winner's curse!) [Thompson et al., 2011; Bush and Moore, 2012]; the higher odds ratios, if confirmed in independent cohorts, may support the general observations that CNVs associated with phenotypes confer a higher effect size than the SNP-based associations [Lin et al., 2011]. Common CNVs identified in this study (online suppl. table 1) need further efforts to identify robust signatures (independent of the $13 \mathrm{CNVs}$ we reported here) through replication studies, as outlined for GWAS study designs.

\section{Putative Functional Relevance of the Identified CNVs}

Recent interest in CNVs is also on their cis-regulatory potential in controlling the expression of nearby genes and therefore the phenotypes. The loss or gain of altered transcription factor binding sites or enhancer elements within the CNV loci may confer the cis-regulation [Schlattl et al., 2011; Haraksingh and Snyder, 2013]. In addition, focus has also been on embedded miRNA, lncRNA, and other small RNA genes transcribed from intergenic and intragenic regions for their obvious roles in post-transcriptional and epigenetic gene regulatory mechanisms [Wu et al., 2012; Meng et al., 2014; Sorensen et al., 2015]. We identified 3 candidate lncRNA genes within or proximal to the CNVs showing association with BC (table 2). Evidence for expression of IncRNA in this region would support the premise that CNVs may in part contribute to the phenotypic variance through the expression of noncoding RNAs regulating protein-coding genes. Independent confirmation of the expression of identified lncRNAs in breast tissues was obtained from the lncRNA expression studies in BC (TANRIC). We compared the normalized counts (output from the database for a queried region) in matched normal and invasive breast tumor tissues ( $\mathrm{n}=105$ pairs from TCGA) for a representative lncRNA (RP11206M11.7; table 2). Expression of this lncRNA was observed in tumor tissues (average of 100 normalized counts) as well as in adjoining normal tissues (average of 52 normalized counts), indicating that the CNVR encompassing this lncRNA is transcriptionally active and is indeed differentially expressed in breast tissues. Further, this lncRNA has been reported as a promising prognostic factor for BC [Meng et al., 2014; Sorensen et al., 2015]. The remaining 2 lncRNAs from chromosome 5 showed low or negligible expression in breast tissues (data not shown). However, we did not identify miRNA genes from the limited number of CNV targets interrogated (listed in table 2).

\section{Discussion}

We identified common CNVs from germline DNA as potential susceptibility variants for BC risk. Thirteen CNVs called by Nexus and Partek showed overlap and were associated with BC. These signatures were identified 
based on concordances for frequencies, effect sizes, and direction (gain or loss) between the 2 algorithms. This is also the first comprehensive study in sporadic BC to mine CNVs utilizing $2 \mathrm{CNV}$ calling algorithms.

Subsets of CNV loci identified (table 2) harbored genes that are known to play a role in BC: (1) NBPF9 and an adjacent gene, $P D E 4 D I P$, are evolutionarily conserved and exhibit CNVRs; these gene loci are mutated in BC and in neuroblastoma [Diskin et al., 2009; O'Bleness et al., 2012; Horvath et al., 2013]; (2) the AUTS2 gene was shown to be prognostic for $\mathrm{BC}$ recurrence and is also an integral member of the PAM50 prognostic gene cluster [Chanrion et al., 2008; Stadler et al., 2012; Nagamani et al., 2013]; (3) ASTN2 gene rearrangements are prognostic in BC [Glessner et al., 2009; Hampton et al., 2009]; and (4) SGCD is known to mediate paclitaxel sensitivity [Conrad et al., 2010b; Eng et al., 2011; Beck et al., 2013; Chettier et al., 2014].

CNVs also potentially harbor cis-regulatory regions and small RNAs and as such are attractive tools [Vaishnavi et al., 2013] to explain the functional relevance for the BC susceptibility variants identified, and the same was demonstrated in this study. A CNV in chromosome 3q25.1 shown to be associated with $\mathrm{BC}$ also harbored lncRNA and showed expression in a tissue-specific manner (table 2). Correlations of expression with the copy number status in breast tumor tissues remain to be determined. Evidence of breast tissue-specific expression and putative functional significance of CNV loci associated with disease phenotypes complement the approach of validating $\mathrm{CNV}$ calls by 2 independent algorithms and lend confidence in the study findings.

In addition to the observed associations with $\mathrm{BC}$ phenotype, the identified CNV regions also were shown to be associated with other diseases (show pleiotropy) such as autism, schizophrenia, multiple sclerosis, and congenital cardiovascular disease [Vandepoele et al., 2005; Glessner et al., 2009; Kao et al., 2010; Stadler et al., 2012; Lalani et al., 2013; Nagamani et al., 2013; Szatkiewicz et al., 2014; Li et al., 2016].

Interestingly, a higher prevalence of autism correlates with a high incidence of in situ BC [Kao et al., 2010]. Autism and incidence of cancers share a common feature, i.e., a high rate of genomic aberrations underlying disease etiology. The above findings are from as of now limited knowledge in recognizing the pleiotropy of gene-disease relationships and underpin the paucity of literature on the shared predisposing mutations, SNPs and CNVs, which play a complex role in conferring disease etiologies.

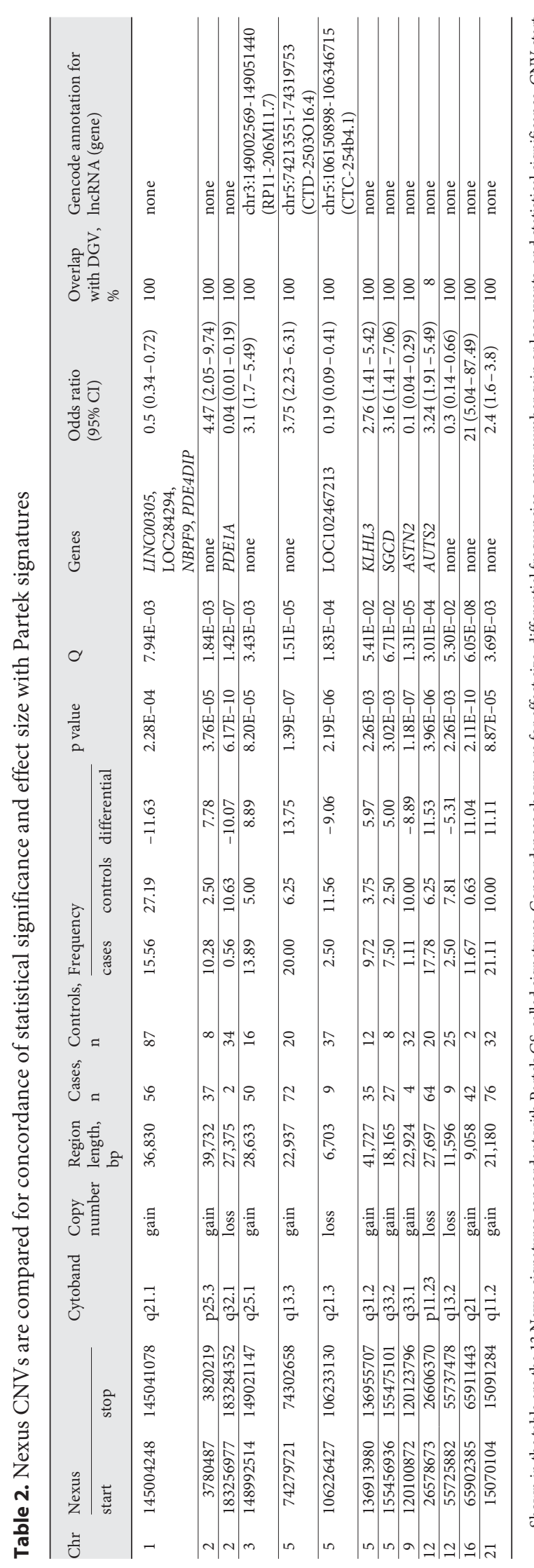

Identification of Germline CNVs for Breast Cancer Susceptibility
Cytogenet Genome Res 2016;149:156-164 DOI: $10.1159 / 000448558$ 
In the GWAS domain, a multi-stage association study design using large cohorts is needed to confer statistical power, and controlling for population stratification is equally important to reduce false positive associations. We controlled for population stratification, applied filters which are stringent for CNV studies, and utilized 2 different CNV calling algorithms to ensure comprehensive detection of CNVs. Some of the reported CNV regions in this study were indeed characterized as disease conferring across a spectrum of phenotypes and therefore provide confidence in our study findings [Butler et al., 2005; Bailey et al., 2008; Diskin et al., 2009; Glessner et al., 2009, 2010; Ehli et al., 2012; Stadler et al., 2012; Lalani et al., 2013; Nagamani et al., 2013; Ukkola-Vuoti et al., 2013; Chettier et al., 2014; Szatkiewicz et al., 2014; Furuya et al., 2015].

Progress in the detection of CNVs as germline determinants of disease risk has been limited and may be ascribed to the suboptimal performances of the segmentation algorithms in ascertaining the breakpoints, genomescale validation of CNVs by qRT-PCR not being costeffective; and limitations on the design of primers for validating complex genomic regions. While comparing 2 algorithms to detect concordant signatures is one approach to narrow down the list of targets for future independent validations, CNVs identified by individual algorithms also deserve attention. Individual algorithms have also been successfully utilized in CNV-GWAS. However, independent cohorts profiled on the same platform or validations of selected CNVs by qRT-PCR are needed for replication of discovery stage findings.

The sample size for cases (BRCA positive or nonBRCA familial) in previous BC CNV studies ranged from 68 to 129 , and for controls from 40 to 128 . The platform technologies also varied (Illumina, Affymetrix Cyto $2.7 \mathrm{M}$ array, Agilent aCGH) as well as $\mathrm{CNV}$ calling algorithms (Agilent, Affymetrix, Nexus, Illumina) [Zhang et al., 2011; Krepischi et al., 2012a; Pylkas et al., 2012; Kuusisto et al., 2013; Masson et al., 2014], making it difficult to compare the signatures across all these studies. Rare CNVs identified to date in familial BC showed little overlap between studies [Krepischi et al., 2012b; Kuusisto et al., 2013; Masson et al., 2014], underscoring the need for large-scale replication studies and further development of robust CNV calling algorithms. The identified common CNV associations described in the present study may help to warrant further work to extend the premise that understanding the heritable nature of $\mathrm{BC}$ susceptibility in populations may also reside outside of SNP-based association-mapping strategies.

\section{Conclusion}

We identified CNVs associated with BC susceptibility; subsets of these CNVs harbored genes known to play a role in BC etiology or prognosis. A comprehensive search for CNVs in BC predisposition or for any phenotype association studies is an iterative exercise as refinements in calling algorithms offering finer resolution of breakpoints for CNV boundaries will likely unravel additional variants in the future. Using 2 or more algorithms for segmentation is a humble start to limit the number of false positive associations. As such, stage 1 of the current CNVGWAS offers putative candidate loci for replication in large and independent cohorts.

\section{Acknowledgements}

The study was supported by funding from the Canadian Breast Cancer Foundation (CBCF)-Prairies/NWT Chapter (to S.D.). The Alberta Cancer Foundation and the CBCF-Prairies/NWT Chapter provided funding for the Alberta Cancer Research Biobank and the CBCF Tumor Bank. We acknowledge the help from the 'Tomorrow Project' for providing the control samples for the association study. The Alberta Cancer Foundation and the Alberta Cancer Prevention and Legacy Fund managed by Alberta InnovatesHealth Solutions provided financial support to the 'Tomorrow Project'. We thank Jennifer Dufour for technical assistance. We thank Ms. Preethi Krishnan for critical reading of the manuscript and suggestions.

\section{Statement of Ethics}

Written informed consent was obtained from all the study participants, and The Alberta Cancer Research Ethics Committee (ACREC) approved the study.

\section{Disclosure Statement}

The authors have no conflicts of interest to declare.

\footnotetext{
References Bailey JA, Kidd JM, Eichler EE: Human copy number polymorphic genes. Cytogenet $\mathrm{Ge}$ nome Res 123:234-243 (2008).

Beck J, Hennecke S, Bornemann-Kolatzki K, Urnovitz HB, Neumann S, et al: Genome aberrations in canine mammary carcinomas and their detection in cell-free plasma DNA. PLoS One 8:e75485 (2013).

Bush WS, Moore JH: Chapter 11: Genome-wide association studies. PLoS Comput Biol 8: e1002822 (2012).
} 
- Butler MG, Dasouki MJ, Zhou XP, Talebizadeh Z, Gonzalez E, Kulkarni H, Bolivar H, Mangano A, Brown M, et al: Subset of individuals with autism spectrum disorders and extreme macrocephaly associated with germline PTEN tumour suppressor gene mutations. J Med Genet 42:318-321 (2005).

Cantsilieris S, Western PS, Baird PN, White SJ: Technical considerations for genotyping multi-allelic copy number variation (CNV), in regions of segmental duplication. BMC Genomics 15:329 (2014).

Castellani CA, Melka MG, Wishart AE, Locke $\mathrm{ME}$, Awamleh Z, et al: Biological relevance of $\mathrm{CNV}$ calling methods using familial relatedness including monozygotic twins. BMC Bioinformatics 15:114 (2014).

Chanrion M, Negre V, Fontaine H, Salvetat N, Bibeau F, et al: A gene expression signature that can predict the recurrence of tamoxifen-treated primary breast cancer. Clin Cancer Res 14: 1744-1752 (2008).

Chettier R, Ward K, Albertsen HM: Endometriosis is associated with rare copy number variants. PLoS One 9:e103968 (2014).

Conrad DF, Hurles ME: The population genetics of structural variation. Nat Genet 39:S30-S36 (2007).

Conrad DF, Bird C, Blackburne B, Lindsay S, Mamanova L, et al: Mutation spectrum revealed by breakpoint sequencing of human germline CNVs. Nat Genet 42:385-391 (2010a).

-Conrad DF, Pinto D, Redon R, Feuk L, Gokcumen $\mathrm{O}$, et al: Origins and functional impact of copy number variation in the human genome. $\mathrm{Na}$ ture 464:704-712 (2010b).

Diskin SJ, Hou C, Glessner JT, Attiyeh EF, Laudenslager $\mathrm{M}$, et al: Copy number variation at 1q21.1 associated with neuroblastoma. $\mathrm{Na}$ ture 459:987-991 (2009).

-Ehli EA, Abdellaoui A, Hu Y, Hottenga JJ, Kattenberg M, et al: De novo and inherited CNVs in $\mathrm{MZ}$ twin pairs selected for discordance and concordance on attention problems. Eur J Hum Genet 20:1037-1043 (2012).

-Eng L, Ibrahim-zada I, Jarjanazi H, Savas S, Meschian M, et al: Bioinformatic analyses identifies novel protein-coding pharmacogenomic markers associated with paclitaxel sensitivity in NCI60 cancer cell lines. BMC Med Genomics 4:18 (2011).

Feuk L, Marshall CR, Wintle RF, Scherer SW: Structural variants: changing the landscape of chromosomes and design of disease studies. Hum Mol Genet 15 Spec No 1:R57-R66 (2006).

Furuya S, Suehiro Y, Namiki Y, Sasaki K: CNVs associated with susceptibility to cancers: a mini-review. J Cancer Therapy 6:413-422 (2015).

-Glessner JT, Wang K, Cai G, Korvatska O, Kim $\mathrm{CE}$, et al: Autism genome-wide copy number variation reveals ubiquitin and neuronal genes. Nature 459:569-573 (2009).

-Glessner JT, Reilly MP, Kim CE, Takahashi N, Albano A, et al: Strong synaptic transmission impact by copy number variations in schizophrenia. Proc Natl Acad Sci USA 107:1058410589 (2010). Sanchez R, et al: The influence of CCL $3 L 1$ gene-containing segmental duplications on HIV-1/AIDS susceptibility. Science 307: 1434-1440 (2005).

Grayson BL, Aune TM: A comparison of genomic copy number calls by Partek Genomics Suite, Genotyping Console and Birdsuite algorithms to quantitative PCR. BioData Min 4: 8 (2011).

Hampton OA, Den Hollander P, Miller CA, Delgado DA, Li J, et al: A sequence-level map of chromosomal breakpoints in the MCF-7 breast cancer cell line yields insights into the evolution of a cancer genome. Genome Res 19:167-177 (2009).

Handsaker RE, Van Doren V, Berman JR, Genovese G, Kashin S, et al: Large multiallelic copy number variations in humans. Nat Genet 47: 296-303 (2015).

Haraksingh RR, Snyder MP: Impacts of variation in the human genome on gene regulation. J Mol Biol 425:3970-3977 (2013).

-Hollox EJ, Hoh BP: Human gene copy number variation and infectious disease. Hum Genet 133:1217-1233 (2014).

Horvath A, Pakala SB, Mudvari P, Reddy SD, Ohshiro K, et al: Novel insights into breast cancer genetic variance through RNA sequencing. Sci Rep 3:2256 (2013).

Kao HT, Buka SL, Kelsey KT, Gruber DF, Porton $\mathrm{B}$ : The correlation between rates of cancer and autism: an exploratory ecological investigation. PLoS One 5:e9372 (2010).

Kidd JM, Cooper GM, Donahue WF, Hayden HS, Sampas N, et al: Mapping and sequencing of structural variation from eight human genomes. Nature 453:56-64 (2008).

Korn JM, Kuruvilla FG, McCarroll SA, Wysoker A, Nemesh J, et al: Integrated genotype calling and association analysis of SNPs, common copy number polymorphisms and rare CNVs. Nat Genet 40:1253-1260 (2008).

Krepischi AC, Achatz MI, Santos EM, Costa SS, Lisboa BC, et al: Germline DNA copy number variation in familial and early-onset breast cancer. Breast Cancer Res 14:R24 (2012a).

Krepischi AC, Pearson PL, Rosenberg C: Germline copy number variations and cancer predisposition. Future Oncol 8:441-450 (2012b).

Kuusisto KM, Akinrinade O, Vihinen M, Kankuri-Tammilehto M, Laasanen SL, Schleutker J: Copy number variation analysis in familial BRCA1/2-negative Finnish breast and ovarian cancer. PLoS One 8:e71802 (2013).

Lalani SR, Shaw C, Wang X, Patel A, Patterson LW, et al: Rare DNA copy number variants in cardiovascular malformations with extracardiac abnormalities. Eur J Hum Genet 21:173181 (2013).

Li J, Han L, Roebuck P, Diao L, Liu L, et al: TANRIC: an interactive open platform to explore the function of lncRNAs in cancer. Cancer Res 75:3728-3737 (2015).
Li J, Cai T, Jiang Y, Chen H, He X, et al: Genes with de novo mutations are shared by four neuropsychiatric disorders discovered from NPdenovo database. Mol Psychiatry 21:290297 (2016).

Li X, Chen X, Hu G, Liu Y, Zhang Z, et al: Combined analysis with copy number variation identifies risk loci in lung cancer. BioMed Res Int 2014:469103 (2014).

Lin P, Hartz SM, Wang JC, Krueger RF, Foroud $\mathrm{TM}$, et al: Copy number variation accuracy in genome-wide association studies. Hum Hered 71:141-147 (2011).

Long J, Delahanty RJ, Li G, Gao YT, Lu W, et al: A common deletion in the APOBEC3 genes and breast cancer risk. J Natl Cancer Inst 105: 573-579 (2013).

MacDonald JR, Ziman R, Yuen RK, Feuk L, Scherer SW: The Database of Genomic Variants: a curated collection of structural variation in the human genome. Nucleic Acids Res 42:D986-992 (2014).

-Masson AL, Talseth-Palmer BA, Evans TJ, Grice DM, Hannan GN, Scott RJ: Expanding the genetic basis of copy number variation in familial breast cancer. Hered Cancer Clin Pract 12: 15 (2014).

McCarroll SA: Copy number variation and human genome maps. Nat Genet 42:365-366 (2010).

McCarroll SA, Altshuler DM: Copy-number variation and association studies of human disease. Nat Genet 39:S37-42 (2007).

Meng J, Li P, Zhang Q, Yang Z, Fu S: A four-long non-coding RNA signature in predicting breast cancer survival. J Exp Clin Cancer Res 33:84 (2014).

-Michailidou K, Hall P, Gonzalez-Neira A, Ghoussaini M, Dennis J, et al: Large-scale genotyping identifies 41 new loci associated with breast cancer risk. Nat Genet 45:353-361, 361e1-2 (2013).

Nagamani SC, Erez A, Ben-Zeev B, Frydman M, Winter $S$, et al: Detection of copy-number variation in AUTS2 gene by targeted exonic array CGH in patients with developmental delay and autistic spectrum disorders. Eur J Hum Genet 21:343-346 (2013).

O’Bleness MS, Dickens CM, Dumas LJ, KehrerSawatzki H, Wyckoff GJ, Sikela JM: Evolutionary history and genome organization of DUF1220 protein domains. G3 2:977-986 (2012).

Park RW, Kim TM, Kasif S, Park PJ: Identification of rare germline copy number variations over-represented in five human cancer types. Mol Cancer 14:25 (2015).

- Purcell S, Neale B, Todd-Brown K, Thomas L, Ferreira MA, et al: PLINK: a tool set for whole-genome association and populationbased linkage analyses. Am J Hum Genet 81: 559-575 (2007).

Pylkas K, Vuorela M, Otsukka M, Kallioniemi A, Jukkola-Vuorinen A, Winqvist R: Rare copy number variants observed in hereditary breast cancer cases disrupt genes in estrogen signaling and TP53 tumor suppression network. PLoS Genet 8:e1002734 (2012).
Identification of Germline CNVs for Breast Cancer Susceptibility
Cytogenet Genome Res 2016;149:156-164 DOI: $10.1159 / 000448558$ 
Sapkota Y, Ghosh S, Lai R, Coe BP, Cass CE, et al: Germline DNA copy number aberrations identified as potential prognostic factors for breast cancer recurrence. PLoS One 8:e53850 (2013a).

Sapkota Y, Yasui Y, Lai R, Sridharan M, Robson PJ, et al: Identification of a breast cancer susceptibility locus at 4q31.22 using a genomewide association study paradigm. PLoS One 8:e62550 (2013b).

-Sapkota Y, Mackey JR, Lai R, Franco-Villalobos C, Lupichuk S, et al: Assessing SNP-SNP interactions among DNA repair, modification and metabolism related pathway genes in breast cancer susceptibility. PLoS One 8: e64896 (2014).

-Schlattl A, Anders S, Waszak SM, Huber W, Korbel JO: Relating CNVs to transcriptome data at fine resolution: assessment of the effect of variant size, type, and overlap with functional regions. Genome Res 21:2004-2013 (2011).

-Sehrawat B, Sridharan M, Ghosh S, Robson P, Cass CE, et al: Potential novel candidate polymorphisms identified in genome-wide association study for breast cancer susceptibility. Hum Genet 130:529-537 (2011).
- Sorensen KP, Thomassen M, Tan Q, Bak M, Cold $\mathrm{S}$, et al: Long non-coding RNA expression profiles predict metastasis in lymph nodenegative breast cancer independently of traditional prognostic markers. Breast Cancer Res 17:55 (2015).

Stacey SN, Manolescu A, Sulem P, Rafnar T, Gudmundsson J, et al: Common variants on chromosomes 2q35 and 16q12 confer susceptibility to estrogen receptor-positive breast cancer. Nat Genet 39:865-869 (2007).

Stacey SN, Manolescu A, Sulem P, Thorlacius S, Gudjonsson SA, et al: Common variants on chromosome 5 p 12 confer susceptibility to estrogen receptor-positive breast cancer. Nat Genet 40:703-706 (2008).

Stadler ZK, Esposito D, Shah S, Vijai J, Yamrom $\mathrm{B}$, et al: Rare de novo germline copy-number variation in testicular cancer. Am J Hum Genet 91:379-383 (2012).

-Szatkiewicz JP, O’Dushlaine C, Chen G, Chambert K, Moran JL, et al: Copy number variation in schizophrenia in Sweden. Mol Psychiatry 19:762-773 (2014).

Thompson JR, Attia J, Minelli C: The meta-analysis of genome-wide association studies. Brief Bioinform 12:259-269 (2011).

-Ukkola-Vuoti L, Kanduri C, Oikkonen J, Buck G, Blancher C, et al: Genome-wide copy number variation analysis in extended families and unrelated individuals characterized for musical aptitude and creativity in music. PLoS One 8:e56356 (2013).
Vaishnavi V, Manikandan M, Tiwary BK, Munirajan AK: Insights on the functional impact of microRNAs present in autism-associated copy number variants. PLoS One 8:e56781 (2013).

-Vandepoele K, Van Roy N, Staes K, Speleman F, van Roy F: A novel gene family NBPF: intricate structure generated by gene duplications during primate evolution. Mol Biol Evol 22: 2265-2274 (2005).

-Wellcome Trust Case Control Consortium, Craddock N, Hurles ME, Cardin N, Pearson RD, et al: Genome-wide association study of CNVs in 16,000 cases of eight common diseases and 3,000 shared controls. Nature 464:713-720 (2010).

Wu X, Zhang D, Li G: Insights into the regulation of human CNV-miRNAs from the view of their target genes. BMC Genomics 13:707 (2012).

Zhang D, Qian Y, Akula N, Alliey-Rodriguez N, Tang J, et al: Accuracy of CNV detection from GWAS data. PLoS One 6:e14511 (2011). 